\title{
Nestin expression is associated with aggressive cutaneous melanoma of the nodular type
}

\author{
Rita G Ladstein ${ }^{1}$, Ingeborg M Bachmann ${ }^{1,2}$, Oddbjørn Straume ${ }^{1,3}$ and Lars A Akslen ${ }^{1,4}$ \\ ${ }^{1}$ Section for Pathology, The Gade Institute, University of Bergen, Bergen, Norway; ${ }^{2}$ Department of \\ Dermatology, Haukeland University Hospital, Bergen, Norway; ${ }^{3}$ Department of Oncology, Haukeland \\ University Hospital, Bergen, Norway and ${ }^{4}$ Department of Pathology, Haukeland University Hospital, \\ Bergen, Norway
}

\begin{abstract}
The intermediate filament nestin, a neural stem-cell marker, is reported to be expressed more strongly in melanomas compared with benign melanocytic lesions, and increasingly expressed in advanced melanoma stages. However, the prognostic impact of nestin on melanoma has not been well elucidated. The aim of the present study was to evaluate the prognostic influence of nestin expression in cutaneous melanoma in comparison with standard clinico-pathologic variables. In a large series of nodular cutaneous melanoma $(n=348)$, nestin expression was assessed by immunohistochemistry using tissue microarray (TMA) sections. For comparison, nestin staining in corresponding metastases as well as in superficial spreading melanomas and benign nevi was also examined. Nestin was expressed to varying degrees in a majority of nodular melanomas (92\%), and was significantly associated with increased tumor thickness, high mitotic count, and the presence of ulceration and tumor necrosis. Also, expression was stronger in the nodular type than in superficial spreading melanomas and benign nevi, but without significant difference when compared with matched metastases from the former. Importantly, strong expression of nestin was significantly associated with reduced survival in multivariate analysis. In conclusion, increased nestin expression was associated with aggressive melanoma features, with independent prognostic impact on multivariate survival analysis when compared with clinico-pathologic factors.

Modern Pathology (2014) 27, 396-401; doi:10.1038/modpathol.2013.151; published online 13 September 2013
\end{abstract}

Keywords: melanoma; nestin; prognosis; survival

Cutaneous malignant melanoma is a potentially aggressive malignancy, with limited therapeutic opportunities in advanced stages. ${ }^{1}$ The incidence is increasing in fairly skinned populations worldwide. $^{2}$ Thus, improved understanding of tumor biology and better prognostication are required as a basis for more precise management.

Nestin is an intermediate filament protein that was first described as a marker of neural progenitor cells during development of the central nervous system. ${ }^{3}$ In addition, expression of nestin has been detected in immature or progenitor cells in nonneural tissues, ${ }^{4,5}$ and nestin is found in association

Correspondence: Dr RG Ladstein, MD or Dr LA Akslen, MD, PhD, Section for Pathology, The Gade Institute, Haukeland University Hospital, University of Bergen, Bergen 5021, Norway. E-mail: rita.ladstein@gades.uib.no or lars.akslen@gades.uib.no Received 4 October 2012; revised 25 July 2013; accepted 25 July 2013; published online 13 September 2013 with injuries and repair in different tissues like the central nervous system and myocardium., ${ }^{4,5}$ In tumors, nestin expression is reported in malignancies of various tissues, and high levels have been correlated with aggressive features in brain tumors, ${ }^{6,7}$ non-small cell lung cancer, ${ }^{8}$ breast cancer, ${ }^{9}$ gastrointestinal stromal tumors and angiosarcoma. ${ }^{10}$ Additionally, nestin has been suggested as a marker of tumor angiogenesis in cancers such as prostate, ${ }^{11}$ breast, ${ }^{12}$ and colorectal cancer. ${ }^{13}$

In cutaneous melanoma, nestin has been reported to be increasingly expressed in more advanced stages, and present only at low levels in benign melanocytic lesions. ${ }^{14,15}$ To the best of our knowledge, a prognostic impact of nestin on cutaneous melanomas has been indicated only in a few studies; ${ }^{16,17}$ however, there has been no assessment of this factor in relation to other characteristics by multivariate analyses. Here, we find that increased nestin expression is independently associated with reduced survival in 
comparison with a panel of clinico-pathologic features in this series of melanomas of the nodular type.

\section{Materials and methods}

\section{Patients}

This patient series consists of consecutive cases of nodular cutaneous melanoma diagnosed at the Department of Pathology, Haukeland University Hospital (Bergen, Norway) during 1981-2008. All cases diagnosed as melanoma of the nodular type, or not otherwise specified, were reviewed by microscopy, and 457 cases were finally included. There was no known history of familial occurrence. The presence of a vertical growth phase and the lack of a radial growth phase, ie, adjacent in situ or microinvasive components, were used as inclusion criteria. Cases with minor secondary involvement of the adjacent epidermis up to three epidermal ridges were included. During this period, the sentinel node procedure was not performed. The first part of this series (1981-1997) has been previously described in detail, ${ }^{18}$ and our material was recently expanded with cases from 1998 to 2008. In addition, 58 paired metastases (local skin, regional lymph nodes, distant) (from 1981 to 1997) were included. The median age was 68 years, 50.1\% were women, and median tumor thickness was $3.6 \mathrm{~mm}$ (range 0.7$44.0 \mathrm{~mm})$.

Complete information on patient survival with time and cause of death was available in all 457 cases. Last date of follow-up was 31 December 2008, and median follow-up time for survivors was 61.5 months (range 0-330 months). During the follow-up period, 147 patients (32\%) died of malignant melanoma, and 100 (22\%) died of other causes.

In addition to this series of nodular melanoma, 32 cases of benign melanocytic nevi (median age 26.6 years) and 20 consecutive cases of invasive superficial spreading melanomas $>1 \mathrm{~mm}$ in thickness (median age 49.0 years; median thickness $1.7 \mathrm{~mm}$; from the period 1981-1983) were included to examine the different categories of melanocytic lesions. Previously reported information on clinico-pathologic characteristics was included for comparison. ${ }^{18-20}$

The Norwegian Data Inspectorate and the Regional Committee for Ethics in Research (Health Region III) (178.05) have approved this project. The study was performed in accordance with the Helsinki Declaration.

\section{Clinico-Pathologic Variables}

The following variables were recorded: date of histologic diagnosis, sex, age at diagnosis, and anatomical site of the primary tumor. The hematoxylin and eosin (H\&E) stained slides were reexamined, and the following histologic features were included in this study: tumor thickness according to Breslow, ${ }^{21}$ the level of invasion according to Clark, ${ }^{22}$ mitotic count, ${ }^{19}$ microscopic tumor ulceration, and tumor necrosis. ${ }^{20}$

\section{Tissue Microarray}

The tissue microarray (TMA) technique has been described and validated in several studies. ${ }^{23-25}$ Three tissue cylinders from representative tumor areas identified on H\&E-stained slides, generally at the suprabasal area of the primary tumors, were punched and mounted into a recipient paraffin block using a custom-made precision instrument (Beecher Instruments, Silver Spring, MD, USA). The diameter of tissue cylinders was $0.6 \mathrm{~mm}$ in the first part of this series (cases 1981-1997), while a diameter of $1.0 \mathrm{~mm}$ was used in the expanded part (cases 1998-2008). Sections of the resulting TMA blocks $(5 \mu \mathrm{m})$ were made by a standard technique.

\section{Immunohistochemistry}

The immunohistochemical staining was performed on thin TMA sections $(5 \mu \mathrm{m})$ of paraffin-embedded archival tissue. The slides were dewaxed with xylene/ethanol before microwave antigen retrieval for $20 \mathrm{~min}$ in Target Retrieval Solution (DAKO S1699) $(\mathrm{pH}=6)$. To prevent endogenous peroxidase activity, the slides were treated with peroxidase block for $5 \mathrm{~min}$. The slides were then incubated for $1 \mathrm{~h}$ at room temperature with a monoclonal mouse nestin antibody (10c2), catalog \# sc-23927 (Santa Cruz Biotechnology, Santa Cruz, CA, USA), diluted 1:200. The staining procedure was performed using the rabbit/mouse EnVision labelled polymer method (Dako, Copenhagen, Denmark), with 3-amino-9ethylcarbazole (AEC) peroxidase as a substrate before brief counterstaining with Dako REAL hematoxylin. Negative controls were obtained by omitting the primary antibody.

Sufficient tumor tissue was available in 348 of the primary tumors; ie, in 100 of the first 202 cases (1981-1997), and in 248 of the 255 cases more recently included (1998-2008). Further, sufficient tissue in TMA sections was available in 44 of the 58 metastases, 17 of the 20 superficial spreading melanomas, and in 30 of the 32 melanocytic nevi.

\section{Evaluation of Staining}

In tumor cells, nestin showed cytoplasmic staining. All positive cases showed varying degrees of cytoplasmic staining. Additionally, a subset of these cases also presented a suggested filamentous and submembranous staining pattern (Figure 1). Tumor cell nuclei were negative in all cases. The endothelial cells were stained, even in cases without expression in tumor cells. 

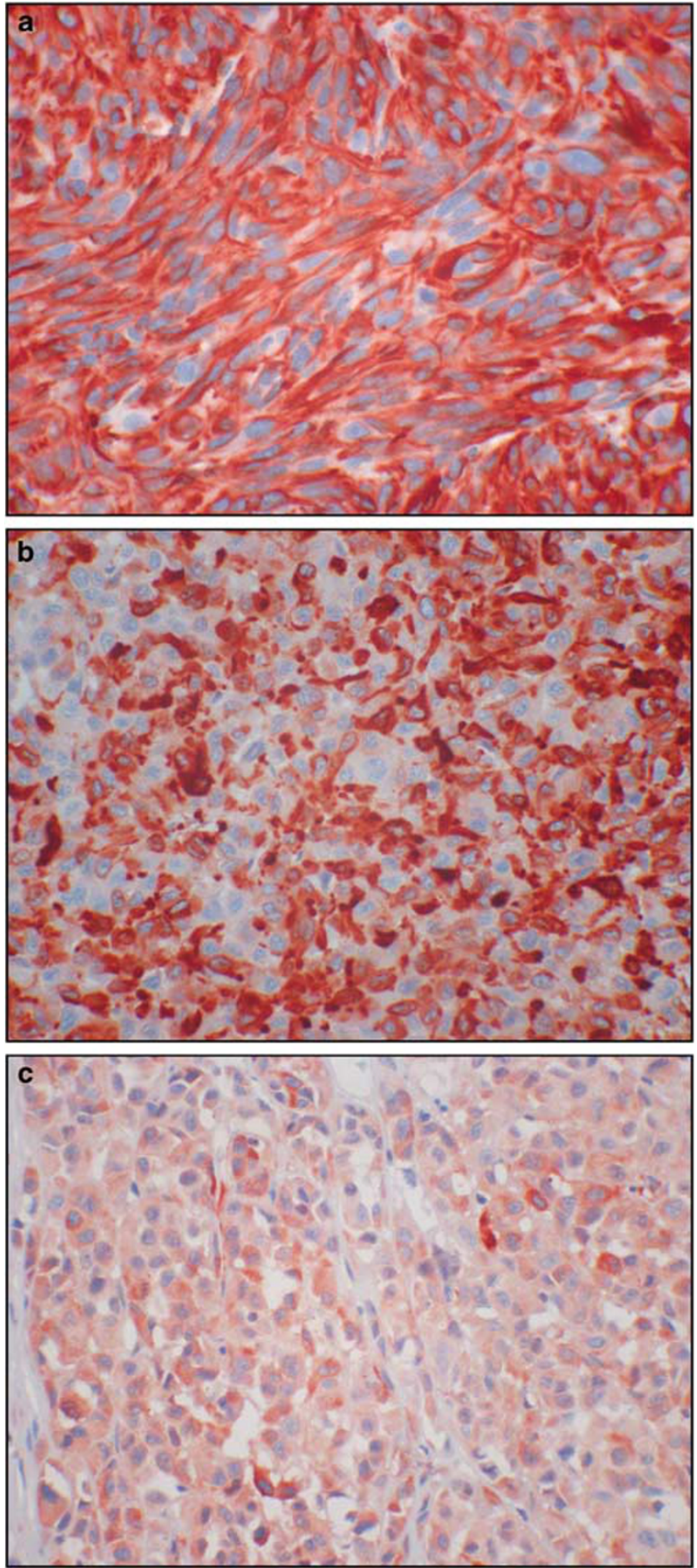

Figure 1 Immunohistochemical staining showing a primary nodular melanoma (a) with strong cytoplasmic and filamentous nestin expression, strong nestin expression in a melanoma metastasis (b), and weaker expression in a superficial spreading melanoma (c).

Immunohistochemical staining of tumor cells was recorded separately for the cytoplasmic and filamentous staining, using a semi-quantitative and subjective grading system, considering both the intensity of staining and the proportion of tumor cells showing unequivocal positive reaction. A staining index (SI) was calculated as a product of staining intensity $(0-3)$ and area of positive tumor cell; $1(<10 \%), 2(10-50 \%)$, and $3(>50 \%) .{ }^{26,27}$ The respective SIs for the two patterns were summarized.

\section{Statistics}

Analyses were performed using the IBM SPSS Statistics 19 (IBM Corporation, Armonk, New York). Associations between different categorical variables were assessed by Pearson's $\chi^{2}$ test or Fisher's exact test. Continuous variables not following the normal distribution were compared between two groups using the Mann-Whitney U-test. McNemar's test was used to compare related samples. The cutoff points for categorization were based on the frequency distribution curves and the size of subgroups as well as the number of events.

Univariate analyses of time to death due to malignant melanoma were performed using the product-limit procedure (Kaplan-Meier method), and differences between categories were estimated by the log-rank test, with date of histologic diagnosis as starting point. Patients who died of causes other than melanoma were censored at the date of death. The influence of co-variates on patient survival was analyzed by the proportional hazards method, and tested by the backward stepwise likelihood ratio (lratio) test. The variables were tested by a log-log plot to determine their ability to be incorporated in multivariate models. All results were considered as significant if $P \leq 0.05$.

\section{Results}

In primary nodular melanoma, nestin was expressed in tumor cells in 319 of the 348 cases recorded $(92 \%)$. The cytoplasmic staining was present in all positive cases, and in addition a filamentous like pattern was expressed in a subset of 273 cases $(78 \%$ of all; $86 \%$ of positive cases). Strong expression of nestin (summarized SI $>3$, by lower quartile) was associated with increased tumor thickness, the presence of tumor ulceration, high mitotic count, and tumor necrosis (Table 1). However, there was no significant correlation between nestin expression and Clark's level of invasion. Strong nestin expression was significantly associated with high mitotic count by PHH3 (Phosphohistone H3) expression $\left(P=0.003, \chi^{2}\right.$ test $)$. The median number of PHH3positive mitotic figures was 31.5 per $\mathrm{mm}^{2}$ in the group with strong nestin expression, compared with median PHH3 count 20.4 per $\mathrm{mm}^{2}$ in the cases with weak nestin $(P=0.001$, Mann-Whitney test). Separate analyses of the two staining patterns gave similar results (not shown).

There was no significant difference in nestin expression in metastases when compared with 
Table 1 Nestin expression in association with histopathologic variables
Table 2 Univariate survival analysis according to nestin expression and clinico-pathologic variables

\begin{tabular}{|c|c|c|c|c|}
\hline \multirow[b]{2}{*}{ Variables } & \multirow[b]{2}{*}{$\mathrm{N}$} & \multicolumn{2}{|c|}{$\begin{array}{c}\text { Estimated survival } \\
\text { rates }(\%)\end{array}$} & \multirow[b]{2}{*}{$\mathrm{P}$-value } \\
\hline & & 5 years & 10 years & \\
\hline Tumor thickness (mm) & & & & $<0.001$ \\
\hline$\leq 2.0$ & 109 & 86 & 81 & \\
\hline $2.1-4.0$ & 164 & 72 & 57 & \\
\hline$>4.0$ & 184 & 54 & 42 & \\
\hline Tumor ulceration & & & & $<0.001$ \\
\hline Absent & 233 & 80 & 71 & \\
\hline Present & 215 & 55 & 43 & \\
\hline Mitotic count (no./mm²) & & & & $<0.001$ \\
\hline$<1.9^{\mathrm{b}}$ & 112 & 84 & 80 & \\
\hline$\geq 1.9$ & 342 & 63 & 50 & \\
\hline Clark's level & & & & $<0.001$ \\
\hline II-IV & 381 & 72 & 62 & \\
\hline V & 75 & 45 & 28 & \\
\hline Tumor necrosis & & & & $<0.001$ \\
\hline Absent & 335 & 75 & 64 & \\
\hline Present & 119 & 47 & 39 & \\
\hline Nestin expression (SI) & & & & 0.002 \\
\hline Low $^{\mathrm{b}}$ & 72 & 85 & 75 & \\
\hline High & 276 & 59 & 45 & \\
\hline Tumor site & & & & 0.007 \\
\hline Non-trunk & 321 & 73 & 62 & \\
\hline Trunk & 132 & 58 & 47 & \\
\hline Age (years) & & & & n.s. \\
\hline$<68^{\mathrm{C}}$ & 225 & 69 & 59 & \\
\hline$\geq 68$ & 232 & 67 & 56 & \\
\hline Gender & & & & 0.002 \\
\hline Female & 229 & 75 & 66 & \\
\hline Male & 228 & 61 & 48 & \\
\hline
\end{tabular}

Abbreviation: SI, staining index.

${ }^{\text {a }}$ Log-rank test.

${ }^{\mathrm{b}}$ Cutoff point lower quartile.

${ }^{\mathrm{C}}$ Cutoff point median value.

\section{Survival Analyses}

Univariate analysis showed significantly reduced survival in cases with strong expression of nestin (summarized SI $>3$ ) in the tumor cells (Figure 2), using a cutoff point equivalent to the lower quartile. In the group of cases with strong expression, the estimated 5-year survival was $59 \%$ and 10 -year survival was $45 \%$. In contrast, 5-year and 10-year survival were 85 and $75 \%$, respectively, in the cases with weak expression of nestin (Table 2).

In multivariate analysis (proportional hazards method), nestin expression was included together with the histopathologic variables tumor thickness, ulceration, mitotic count, Clark's level of invasion, tumor necrosis, as well as anatomical site and gender. In the final model, all variables remained, except mitotic count and ulceration (Table 3). Tumor median $\mathrm{SI}=2.0)\left(P<0.001 ; \chi^{2}\right.$ test $)$. 
Table 3 Multivariate survival analysis (Cox's proportional hazards method), with final model after inclusion of tumor thickness, ulceration, mitotic count, Clark's level, tumor necrosis, nestin expression, tumor site, and gender $(n=339)$

\begin{tabular}{|c|c|c|c|c|}
\hline Variable & $\mathrm{N}$ & $H R$ & $95 \% C I$ & $\mathrm{P}$-value ${ }^{a}$ \\
\hline Tumor thickness (mm) & & & & 0.001 \\
\hline$\leq 2.0$ & 69 & 1 & & \\
\hline $2.1-4.0$ & 122 & 4.8 & $1.71-13.58$ & 0.003 \\
\hline$>4.0$ & 148 & 4.8 & $1.65-13.78$ & 0.004 \\
\hline Clark's level & & & & 0.002 \\
\hline II-IV & 278 & 1 & & \\
\hline $\mathrm{V}$ & 61 & 2.2 & $1.35-3.67$ & \\
\hline Tumor necrosis & & & & 0.013 \\
\hline Absent & 244 & 1 & & \\
\hline Present & 95 & 1.7 & $1.13-2.61$ & \\
\hline Nestin expression (SI) & & & & 0.045 \\
\hline Low ${ }^{b}$ & 70 & 1 & & \\
\hline High & 269 & 1.8 & $1.0-3.17$ & \\
\hline Tumor site & & & & 0.075 \\
\hline Non-trunk & 236 & 1 & & \\
\hline Trunk & 103 & 1.5 & $0.97-2.29$ & \\
\hline Gender & & & & 0.008 \\
\hline Female & 157 & 1 & & \\
\hline Male & 182 & 1.7 & $1.15-2.63$ & \\
\hline
\end{tabular}

Abbreviations: HR, Hazard ratio; CI, confidence interval; SI, staining index.

${ }^{a}$ Likelihood ratio or Wald test.

${ }^{\mathrm{b}}$ Cutoff point lower quartile.

thickness, Clark's level of invasion, tumor necrosis, gender, and nestin expression were all independently significant.

\section{Discussion}

In this study of cutaneous melanoma of the nodular type, strong expression of nestin was associated with markers of aggressive tumors and reduced survival by multivariate analyses. To the best of our knowledge, independent prognostic importance of nestin has not been previously shown in these tumors. By immunohistochemistry, the prognostic impact has been indicated for localized melanoma in two small studies. ${ }^{16,17}$ In a group of stage I-II melanomas $(n=73)$, significantly reduced survival was found in cases with increased nestin expression in both tumor cells and endothelial cells. ${ }^{16}$ In another study of 78 cases, there was significantly worse prognosis in localized melanoma expressing nestin. ${ }^{17}$ The 5 -year survival was $>80 \%$ in nestinnegative cases of all stages. ${ }^{17}$ These studies did not report multivariate survival analyses to compare prognostic impact of nestin in relation to other features. ${ }^{16,17}$ In support of these observations, nestin expression has also been found on circulating melanoma cells, and increased proportion of nestinpositive cells was related to worse prognosis. ${ }^{28}$

The level of nestin expression has been reported to increase during tumor development, with higher levels in melanomas than in dysplastic or common nevi, and also with increased levels in metastases compared with primary tumors. ${ }^{14,15}$ The elevated levels in malignant compared with benign melanocytic lesions have been found statistically insignificant by others. ${ }^{29}$ Still, nestin expression differentiated between nodal melanoma metastases and nodal melanocytic nevi, by strong expression in the former, in a recent study. ${ }^{30}$ In our study, there was no significant difference in nestin expression between primary nodular melanomas and their matched metastases. Similar finding has been reported in smaller studies when comparing nestin in the subgroup of nodular melanoma and melanoma metastases. ${ }^{31}$ However, nestin expression was significantly stronger in nodular melanomas when compared with small subsets of superficial melanomas and benign nevi, indicating an importance of this marker for tumor progression. This is in line with other studies. ${ }^{31}$ The rather weak staining of nestin in benign nevi, taken together with its known expression in immature and pluripotent cells, might support the understanding of nestin as a marker of dedifferentiation during tumor evolution. ${ }^{15,32}$ In our study, this finding is also supported by significant associations between high nestin expression and increased tumor cell proliferation as well as tumor necrosis, both markers found in dedifferentiation and more highgrade malignant tumors.

In conclusion, increased nestin expression was associated with aggressive melanoma features and reduced survival in this series of nodular cutaneous melanoma.

\section{Acknowledgements}

We thank Gerd Lillian Hallseth, Bendik Nordanger, and Randi Hope Lavik for excellent technical assistance. Funding sources were University of Bergen, Norwegian Cancer Society, Norwegian Research Council, and Helse Vest HF.

\section{Disclosure/conflict of interest}

The authors declare no conflict of interest.

\section{References}

1 Garbe C, Eigentler TK, Keilholz U, et al. Systematic review of medical treatment in melanoma: current status and future prospects. Oncologist 2011;16:5-24.

2 Garbe C, Leiter U. Melanoma epidemiology and trends. Clin Dermatol 2009;27:3-9. 
3 Lendahl U, Zimmerman LB, McKay RD. CNS stem cells express a new class of intermediate filament protein. Cell 1990;60:585-595.

4 Wiese C, Rolletschek A, Kania G, et al. Nestin expression-a property of multi-lineage progenitor cells? Cell Mol Life Sci 2004;61:2510-2522.

5 Ishiwata T, Matsuda Y, Naito Z. Nestin in gastrointestinal and other cancers: effects on cells and tumor angiogenesis. World J Gastroenterol 2011;17: 409-418.

6 Milde T, Hielscher T, Witt $\mathrm{H}$, et al. Nestin expression identifies ependymoma patients with poor outcome. Brain Pathol 2012;22:848-860.

7 Zhang M, Song T, Yang L, et al. Nestin and CD133: valuable stem cell-specific markers for determining clinical outcome of glioma patients. J Exp Clin Cancer Res 2008;27:85.

8 Ryuge S, Sato Y, Wang GQ, et al. Prognostic significance of nestin expression in resected non-small cell lung cancer. Chest 2011;139:862-869.

9 Liu C, Chen B, Zhu J, et al. Clinical implications for nestin protein expression in breast cancer. Cancer Sci 2010;101:815-819.

10 Yang $\mathrm{XH}, \mathrm{Wu} \mathrm{QL}, \mathrm{Yu} \mathrm{XB}$, et al. Nestin expression in different tumours and its relevance to malignant grade. J Clin Pathol 2008;61:467-473.

11 Gravdal K, Halvorsen OJ, Haukaas SA, et al. Proliferation of immature tumor vessels is a novel marker of clinical progression in prostate cancer. Cancer Res 2009;69:4708-4715.

12 Kruger K, Stefansson IM, Collett K, et al. Microvessel proliferation by co-expression of endothelial nestin and Ki-67 is associated with a basal-like phenotype and aggressive features in breast cancer. Breast 2012;22:282-288.

13 Teranishi N, Naito Z, Ishiwata T, et al. Identification of neovasculature using nestin in colorectal cancer. Int J Oncol 2007;30:593-603.

14 Klein WM, Wu BP, Zhao S, et al. Increased expression of stem cell markers in malignant melanoma. Mod Pathol 2007;20:102-107.

15 Florenes VA, Holm R, Myklebost O, et al. Expression of the neuroectodermal intermediate filament nestin in human melanomas. Cancer Res 1994;54:354-356.

16 Piras F, Perra MT, Murtas D, et al. The stem cell marker nestin predicts poor prognosis in human melanoma. Oncol Rep 2010;23:17-24.

17 Tanabe K, Amoh Y, Kanoh M, et al. Prognostic significance of the hair follicle stem cell marker nestin in patients with malignant melanoma. Eur J Dermatol 2010;20:283-288.

18 Straume O, Sviland L, Akslen LA. Loss of nuclear p16 protein expression correlates with increased tumor cell proliferation (Ki-67) and poor prognosis in patients with vertical growth phase melanoma. Clin Cancer Res 2000;6:1845-1853.

19 Ladstein RG, Bachmann IM, Straume O, et al. Prognostic importance of the mitotic marker phosphohistone h3 in cutaneous nodular melanoma. J Invest Dermatol 2012;132:1247-1252.

20 Ladstein RG, Bachmann IM, Straume O, et al. Tumor necrosis is a prognostic factor in thick cutaneous melanoma. Am J Surg Pathol 2012;36:1477-1482.

21 Breslow A. Thickness, cross-sectional areas and depth of invasion in the prognosis of cutaneous melanoma. Ann Surg 1970;172:902-908.

22 Clark WH Jr, From L, Bernardino EA, et al. The histogenesis and biologic behavior of primary human malignant melanomas of the skin. Cancer Res 1969;29:705-727.

23 Kononen J, Bubendorf L, Kallioniemi A, et al. Tissue microarrays for high-throughput molecular profiling of tumor specimens. Nat Med 1998;4:844-847.

24 Nocito A, Bubendorf L, Tinner EM, et al. Microarrays of bladder cancer tissue are highly representative of proliferation index and histological grade. J Pathol 2001;194:349-357.

25 Straume O, Akslen LA. Importance of vascular phenotype by basic fibroblast growth factor, and influence of the angiogenic factors basic fibroblast growth factor/fibroblast growth factor receptor-1 and ephrin-A1/EphA2 on melanoma progression. Am J Pathol 2002;160:1009-1019.

26 Aas T, Borresen AL, Geisler S, et al. Specific P53 mutations are associated with de novo resistance to doxorubicin in breast cancer patients. Nat Med 1996; 2:811-814.

27 Straume O, Akslen LA. Alterations and prognostic significance of p16 and p53 protein expression in subgroups of cutaneous melanoma. Int $\mathrm{J}$ Cancer 1997;74:535-539.

28 Fusi A, Reichelt U, Busse A, et al. Expression of the stem cell markers nestin and CD133 on circulating melanoma cells. J Invest Dermatol 2011;131:487-494.

29 Setia N, Abbas O, Sousa Y, et al. Profiling of ABC transporters ABCB5, ABCF2 and nestin-positive stem cells in nevi, in situ and invasive melanoma. Mod Pathol 2012;25:1169-1175.

30 Chen PL, Chen WS, Li J, et al. Diagnostic utility of neural stem and progenitor cell markers nestin and SOX2 in distinguishing nodal melanocytic nevi from metastatic melanomas. Mod Pathol 2012;26:44-53.

31 Brychtova S, Fiuraskova M, Hlobilkova A, et al. Nestin expression in cutaneous melanomas and melanocytic nevi. J Cutan Pathol 2007;34:370-375.

32 Ehrmann J, Kolar Z, Mokry J. Nestin as a diagnostic and prognostic marker: immunohistochemical analysis of its expression in different tumours. J Clin Pathol 2005;58:222-223. 